\title{
Air Pollution Analysis using Ontologies and Regression Models
}

\author{
Parul Choudhary \\ Post Graduate of Computer Science \& Engineering \\ JSSATE \\ Noida, India
}

\author{
Dr. Jyoti Gautam \\ HOD \& Associate Professor (CSE) \\ JSSATE \\ Noida, India
}

\begin{abstract}
Rapidly throughout the world economy, "the expansive Web" in the "world" explosive growth, rapidly growing market characterized by short product cycles exists and the demand for increased flexibility as well as the extensive use of a new data vision managed data society. A new socio-economic system that relies more and more on movement and allocation results in data whose daily existence, refinement, economy and adjust the exchange industry. Cooperative Engineering Co operation and multi -disciplinary installed on people's cooperation is a good example. Semantic Web is a new form of Web content that is meaningful to computers and additional approved another example. Communication, vision sharing and exchanging data Society's are new commercial bet. Urban air pollution modeling and data processing techniques need elevated Association. Artificial intelligence in countless ways and breakthrough technologies can solve environmental problems from uneven offers. A method for data to formal ontology means a true meaning and lack of ambiguity to allow us to portray memo. In this work we survey regression model for ontologies and air pollution.
\end{abstract}

Keywords-Ontologies; Air pollution Analysis; Regression Models; Linear Regression

\section{INTRODUCTION}

The term "ontology" Data Science and research in 1990 across countless man-made intelligence (AI) research community has to. AI researchers have adopted the term "ontology" is usually depicted in a scheme to code they even (from the view point of the computational society) should be a fair representative of the world.

Ontology is a fair; a public explanation of the concept is clear. A "concept" of an event, the event is prepared by identifying the relevant considerations of a hypothetical idea."Formal" way of ontology languages are always involved in the use of machine-readable form. For eg., in the areas of health, disease and symptom of ideas, they are the relationship between cause and not just a nuisance that can cause a disease.

An ontology is a "share" concept that the use of ontologies for the target group aimed to embody the vision of consensus. Ideally ontology terms for its use independently and in a way that the public may be universal, but useful functions arrest uneven and unequal representation of vision calls for an ontology uses.

Meaning how ontologies are helpful in data recovery?
Budding accelerated digitization processes and globally relevant databases that are transpiring in the current year, the focus of the problems of data is modified.

This matter is now the subject of a precise vision to find the data and commands, but only the most relevant agents is difficult to choose from the huge piles of data. Such a syntactic conventional engine, hunting for keywords and its abundance by data elements in order to explore the processes in place is used. A situation that relevant data, including but not a precise data object is different because it uses words to portray - These methods suffer from setbacks such as terminology inconsistency and "contrast" that irrelevant data because of the similarities in wording has been taken.

Recently, however, is moving in a new way - meaning approach. Data about data - - order an additional satisfactory method for data retrieval and exploration of those "need to answer this is to use meta data.

Ontologies are useful in two ways in improving the process:

1) It allows to abstract the information and represent it explicitly- highlighting the concepts and relations and not the words used to describe them. [1]

2) Ontologies can possess inference functions, allowing more intelligent retrieval. For example, a "basketball player" is also a "professional athlete", and an Ontology that defines the relations between these concepts can retrieve one when the other is queried.

\section{WHAT IS ONTOLOGY?}

"Although the ontology is currently a fashionable word, there is no agreement on the precise meaning of the term: it is reported that the main purpose of the ontology is to regulate the meaning of words, the term" ontology "is not clearly defined humorous "and" concerning AI (Artificial Intelligence) to produce a lot of controversy in the discussion "feel. F karma, vision and multimedia ontology engineering request finds applicability in many areas, and each one of them has its own meaning.

\section{1) A bit of etymology and philosophy.}

The term "ontology" last a very long time in philosophy, starting with the works of Aristotle. As being "science", from the Greek "ontos" This way both speech and reason for being 
and "Logo" is described. From the point of thinking about the event, an additional current philosophy is to start with German theorists of the 19th century; the existence of ontology is a systematic report. This idea does not mean different things back (phenomenological approach) are going to be and despite the appearance, these are two ways that cannot be included (phenomenological reduction concerning contemplate). To keep in mind, the larger epistemological sense can help us to understand what must be ontology. Vision, language and reasoning: As a matter of fact, one can maintain three dimensions. In other words: to clear speech about the world, to manipulate our understanding and representation throughout the world to understand the concept.

\section{2) The knowledge engineering point of view.}

Multimedia and vision engineering sectors nineties instead of a systematic nature, a spiritual way of being able to focus more on early reports of the presence of 1 used the word ontology. As a matter of fact, man-made system of intelligence, what exists is a declarative language that can be embedded in. Ontology next how objects, ideas and connections in a short period of interest are agreeing to continue to embody a clear specification is appropriate. Gruber shouted ideas and connections that it is an agent or agents can continue for a description of the area (a program like a reasonable specification) that "the concept of a specification is". The definition of terms used in engineering include semantic web, we can say that to begin again: "An ontology is a computer-readable language to express ideas in a common description of the connection." Fig. 1 is an example of general higher ontology.

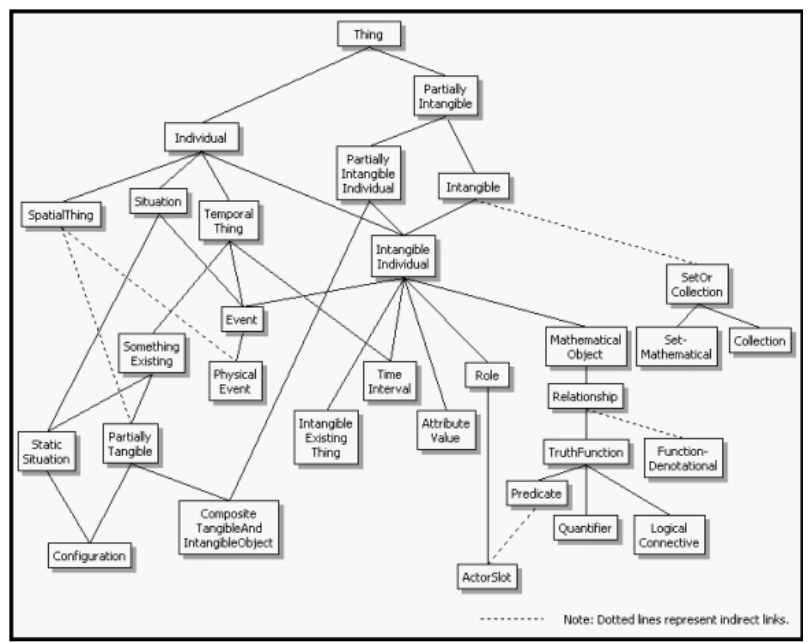

Fig. 1. The Upper Ontology

\section{WHAT IS ONTOLOGY FOR?}

"What is vital is what ontology is for" (T.R Gruber). As a matter of fact, the ontology's necessities depend on the intention of the final application. The main goal of an ontology is to enable contact and vision allocating amid computer arrangements by seizing a public understanding of words that can be utilized by humans and programs. We can next recognize disparate groups of uses for ontologies:

- communication amid people and organizations;
- inter -operability (communication) amid arrangements, including enterprise modeling and multi agent arrangements;

- system engineering, for specification, reliability and reusability;

- knowledge association like data retrieval, document association, vision center systems;

- natural speech treatment for semantic research, lexical construction;

In connection with all requests and Internet electronic transactions and the semantic web, we are well past the one that shows the trend of the real ontology allow inadequate word to say confronting the increasing use of the web, additional human and computer programs to interact with the demand for data on websites. Described as an extension of the current one, a new form of web content was necessary. "Semantic web is a representation of the globe vast web of data".

(W3C's definition): Semantic Web on Resource Description Framework (RDF) for words, XML for syntax and URIs for shouting has been established.

\section{AIR POLLUTION}

Air pollution and prepare for every metropolis outstanding importance for nature is an environmental shock. And in the industry spans the development of motor-car traffic over the past year has been the severity of the situation. Numerous authors and institutions solve the problem of air pollution in the disturbances. Emissions of pollutants that affect air quality, air quality, very much. Lung infections and a high degree of air pollution, with spans of respiratory diseases in the population decline of the sensitivity of the air quality reasons. Car density and air pollution caused by the industry, cars and industry emissions, depending on the type of uneven geographical location, such as temperature, wind conditions, according to the weather conditions, and complementary factors. For the calculation of air pollution emissions, the dense monitoring systems are working in many areas of city. Intended area of monitoring indoor air quality is to tell about the powers accountable. Surveillance data from shock and such small words to solve the contamination level forecasting, emergency response measures, deciding to eliminate air pollution in the form of work is used by experts.

Decision-making has been installed on quantitative data and measurements and observations, presented data concerning the frequency and size of the change and weather standards are maintained from the beginning is consolidated.

I for cooperation in air quality decision support to offer an unfailing, a mathematical model has to be designed, environment protection and air pollution (NO, NO2, mist, $\mathrm{SO} 2$, etc.) between connection established. Obviously, there are unforeseen factors that could impact the degree of air pollution are a lot of, and it is certain that the institution due to a cut or an increase of air pollutants is difficult pointer. Install heavy numerical modeling methods and computational resources when running complex data that often are not 
attainable need to rush. AI methods, expert systems and knowledge-based methods employ the way in this area are not enthusing. The successes of these methods are used in various fields of science. Environmental Law Association and the Association of air quality models and data processing components and modules encompass blow to solve. A knowledge-based numerical model is another route proposal; a vision center is an inference engine that can deal with uncertainty in terms of use by many original features. An ontology way, employing a consistent, coherent and nonredundant central vision can be estimated.

\section{URBAN AIR QUALITY MONITORING}

Wise accurate environmental data, uneven metropolitan locations, the resort affords elevated alarm system possible scenarios and to advocate appropriate countermeasures should notice. Mobility is possible to exploit the inherent web nodes, consequently, a low cost environmental monitoring system with high spatial coverage to apply.

With a vehicular sensor web in potential environmental data collectable, air quality plays a second role. It's really a concern in modern cities because of air pollution effect on population and environment is an important encounter.

Local factors, the city's terms, and conditions hot spot: the air quality in the city spread is the result of three factors. In rural spans, pollution levels with medium to long-distance voyaging complementary areas of air pollutants from the crowd mainly depend on transportation. Emerging compression levels are significantly less in these areas. The city spans, the air contamination such warming settings and lighting off, region and private vehicular transport, or assembly activities as human hobby, is related to the set. City pollution accordingly human passion, the topography, and the innate micrometeorology, spatial varies as it is reasonable to anticipate.

The importance of this topic is so high that the Directive 96/62 / EC, establishes the principles that Frank is a public strategy and delineate or to circumvent the order, cut close to the ambient air quality targets in the European is manipulated by the commission on the human condition and nature of harmful consequences, Associate States assess ambient air standard in the region to inform and enhance the quality of air, while it is undesirable. Air pollution is usually very good web is monitored by permanent stations. A tracking station just a huge scope of pollution standard research tool can calculate work. However, continuous monitoring stations repeatedly so as to measure the surrounding background concentrations or possible hotspot areas are assigned which normally are in addition to the countless kilometers. In addition, the purchase and maintenance of such a heavy price to limit the number of capacities manipulating the dimensional resolution of the pollution map is emerging. In fact, a stable sensor capable of spatial coverage scope is manipulated, so it's a huge interest in a vast number of detectors for monitoring period should seize. These failures, we advocate a profitable and feasible channel sensor networks win. If requested efficiently, a VSN can offer a wide dimensional coverage, and saw a better quality of the characteristics. Instead of employing fixed sensors, detectors in cars or transport services sector can be installed on vehicles.
Sensors secured on advancing vehicles periodically track the air standard and send the taken data to a central storage system. Though, there is a trade-off for this gain in dimensional coverage. Time-related coverage of detected information in a particular locale will be less contrasted to fixed sensors readings as a feature will be measured in the alike locale only when the vehicle will cross once more that point. This lack of time-related coverage can be grasped by rising web nodes density and so climbing sensors on extra vehicles, or allocating sensors on area buses, so that environmental features can be tracked unceasingly alongside their routes. One more setback that ought to not be underrated concerns degraded measurements inside sensor networks. Sensor nodes could sporadically produce wrong measurements due to battery exhaustion, dust on sensors, tampering and supplementary causes. Amid the countless mathematical methods and algorithms in works, suitable instruments to preprocess such gathered data are Bayesian Networks.

In the subsequent serving we debate a little continuing air contamination monitoring period bestowing a brief overview of the state of the fine art.

\section{ONTOLOGICAL APPROACH}

Urban air corruption affiliation needs raised demonstrating and information preparing procedures. Synthetic mind gives incalculable techniques and advances that can resolve usefully dissimilar ecological issues. AI techniques present increases above additional set up numeric demonstrating ways that need substantial processing assets and interest as info convoluted data, much of the time not easily accessible. It is critical to settle on choices in natural security affiliation, so a multispecialist game plan (MAS) way may be asked for better arrangement. A multi-specialist game plan is a web of interactive media operators that conveys to determine mishaps that are surpassing the individual limits or vision of each and every misfortune solver. A specialist can be a physical or neighboring presence that can deed autonomously and has mastery to finish its points and slant. The learning of smart specialists and multi-operator game plans gives the interactive media preparation to the execution of disseminated environment courses of action that can track and control the ecological standard.

In our setting, the vehicular sensor web can be demonstrated as a multi-operator framework made by a set out of astute substances. Each and every vehicle has a bit of running media specialists that screen and analyze the advancement of natural information like air quality, and report to a manager operator if somewhat basic mishap can happen. As per Chomsky speculations, information, assembled by sensors introduced on vehicles, must be epitomized in an appropriate strategy so that keen choice operators can understandable and reason on it. A technique to formalize information is the significance of a right cosmology that grants us to depict reminders close by importance and lacking uncertainty. One of the additional open points in developing ontologisms is apportioning open comprehension of the development of information in the midst of individuals or interactive media specialists. The contact in the midst of specialists depends by and large on the reception of a 
conceptualization, that is, a legitimate representation of the truth of a particular circumstance, so oncologic pace is straight to the point in our course of action, keeping in mind the end goal to portray an open vocabulary for scientists who interest to allot information around there, yet also for learning based arrangements outlining that inquiries and attestations are traded in the midst of operators. The utilization of distinguishing components and remote sensor networks is rising, so a rising volume of heterogeneous information, information organizations, and estimation methods is produced. The ontological perfect gives a technique to deal with the sensors and the convoying volume of created information. It can moreover be used to accept sensor readings and to deal with imperfect sensor information as portrayed in, in that the W3C Semantic Sensor Web Incubator group (SSNXG) depicted OWL 2 cosmology to outline the aptitudes and properties of sensors, the deed of identifying and the developing perceptions.

So as to formalize air quality contemplations, philosophy could be used to development air sullying zone ideas. Since a standout amongst the most crucial philosophy elements is its reusability, we accepted to development our vision range as per AIR POLLUTION Ontology. This cosmology is committed to air defilement examination and mastery, and has been genuinely used in Air Pollution.

Urban air pollution organized information will be justifiable and process capable by specialists whose point is to screen and moderate defilement results crosswise over different sorts of uses. Case in point, joining 3D city models, meteorological elements and air tainting information, it is plausible to guesstimate the nature of air in a little city zone. One additionally fascinating solicitation may be street activity administration. Utilizing the information enriched by natural and street metaphysics, it ought to be likely to build up somewhat smart mixed media specialist that can set a proposed most extreme velocity for a vehicle, or effect activity lights keeping in mind the end goal to get a handle on the quantity of vehicles on the streets of a specific city zone. These activities may provide for scatter meteorological poisons in this way cutting their pressure in building appended ranges.

\section{BUILDING OF ONTOLOGY FOR AIR POLLUTION CONTROL}

Making metaphysics is not a paltry issue. It needs not simply aptitudes in information advances but rather also profound vision in the demonstrated space. The strategy of vision course of action advancement is organized in several models that must be made. On the "setting" level of reflection three models is proposed: Organizational perfect, Task perfect and Agent's model. The authoritative perfect portrays the relationship close by the objective to see the mishaps and chances of vision administration. The Task perfect epitomizes errand that are gave inside the association. An undertaking is whatever that must be given by a specialist. The operator perfect portrays all specialists - agents of assignments - their demonstrations, capabilities, abilities, and confinements. Metaphysics progress systems help making ontologies in grouped region arranged applications. Endless procedures have been industrialized so as to formalize making Ontologies for assembling or supplementary applications. Despite the fact that cosmology progresses systems are not develop bounty, they can be useful in developing philosophy set up vision courses of action.

\section{RELATED WORKS}

J., Fenger, et. al. (1999) [1] In this paper, following 1950 the world populace has dramatically increased, and the worldwide number of autos has expanded by a component of 10. In the same time frame the part of individuals living in urban ranges has expanded by a component of 4 . In year 2000 this will add up to about portion of the world populace. Around 20 urban areas will each have populaces above 10 million individuals. Seen over longer periods, contamination in real urban communities tends to increment amid the developed stage, they go through a most extreme and are of course diminished, as reduction methodologies are created. In the industrialized western world urban air contamination is in a few regards in the last stage with successfully diminished levels of sulfur dioxide and residue. In late decades in any case, the expanding movement has changed the consideration regarding nitrogen oxides, natural mixes and little particles. In a few urban areas photochemical air contamination is a critical urban issue, however in the northern piece of Europe it is a huge scale wonder, with ozone levels in urban avenues being ordinarily lower than in rustic territories. Urban communities in Eastern Europe have been (and by and large still are) intensely dirtied. After the late political change, trailed by an impermanent subsidence and a consequent presentation of new innovations, the circumstance seems to make strides. Be that as it may, the rising number of private autos is a rising issue. In most creating nations the fast urbanization has so far brought about uncontrolled development and falling apart environment. Air contamination levels are here as yet ascending on numerous fronts.

Noboru, Yamazoe, Go Sakai et. al. (2003) [2] In this paper, semiconductor gas sensors use permeable polycrystalline resistors made of semiconducting oxides. The working guideline includes the receptor capacity played by the surface of every oxide grain and the transducer capacity played by every grain limit. Furthermore, the utility variable of the detecting body additionally participates in deciding the gas reaction. In this manner, the ideas of sensor outline are controlled by considering each of these three key variables. The necessities are choice of a base oxide with high versatility of conduction electrons and attractive steadiness (transducer capacity), choice of an outside receptor which improves surface responses or adsorption of target gas (receptor capacity), and creation of a very permeable, meager detecting body (utility component). Late advance in sensor plan taking into account these elements is depicted.

Mihaela. Oprea et. al. (2005) [3] In this paper, air contamination control in urban districts is one of the fundamental bearings of exploration in the ecological sciences. For every area the contamination causes and contamination scattering are distinctive, contingent upon the 
modern movement, on vehicles activity, on residential sources et cetera, and also on the topographical area, temperature of the air, velocity and heading of the wind, and other climate elements. A few scientific models are utilized for the portrayal of the connections between natural insurance and meteorological elements. An option way to deal with the numerical models is an information based methodology that incorporates numerous wellsprings of learning in an information base. The paper portrays a contextual investigation of learning displaying in an air contamination control choice emotionally supportive network that utilizations DIAGNOZA_MEDIU, a model master framework committed to air contamination examination and control in urban areas. We have created metaphysics, AIR_POLLUTION, for the application area. A few AI procedures were utilized as a part of the learning demonstrating process. A counterfeit neural system gives prescient information to the actualities base, and a part of the principles from the tenet base are removed by utilizing inductive learning.

Michael, Blaschke, Thomas Tilleet. al. (2006) [4] In this paper, microelectromechanical-framework (MEMS) metaloxide gas sensors have achieved a full grown stage, which makes mass business sector applications in the car region conceivable. As opposed to the officially settled fold control framework, which controls the entrance of (ignition) gasses from outside the vehicle to the auto lodge, the framework contemplated here distinguishes scent occasions made inside the auto lodge. The occasions under study have been tobacco smoke, fast-food smell, excrement, and bio effluents (tooting). As the reference can't be a "straightforward logical estimation," a human test board for evaluating the hedonic impact on a scale from 0 to 5 is utilized as reference. The specialized framework is a MEMS metal-oxide-sensor cluster comprising of three unique sensors. The information assessment approach utilized here is consolidating the humantactile information and the MEMS sensor information. The assignment is performed by the blend of two free calculations, where one is identified with the standardized conductance and the other to flag change. Utilizing a consolidated methodology has the favorable position that "false" occasions are smothered. After the calculation was effectively exchanged onto a microcontroller, genuine information were recorded and grouped. A few functional cases are given in this paper. The general gas-sensor framework achieves great agreement with the human-tactile impression, which is spoken to via air quality levels. This empowers the outline of an interest controlled ventilation framework.

H. K. Eminir, Hala Abdel-Galil et. al. (2006) [5] In this paper, the absence of natural information is a typical component of numerous creating nations. This is truth in Egypt, where air quality is starting to be efficiently observed in a few spots of the nation. To defeat these issues, the requirement for exact assessments of air quality levels turns out to be always vital. To accomplish such forecast errands, the utilization of fake neural system (ANN) is viewed as a practical procedure better than conventional factual techniques. In this paper, ANN prepared with a back spread calculation is utilized to appraise the surely understood poisons, from promptly discernible neighborhood meteorological information. The outcomes demonstrate that the ANN model anticipated air contamination focuses with great exactness of roughly $96 \%$.

Uichin, Lee, Biao Zhou, et. al. (2006) [6] In this paper, vehicular sensor systems are developing as another system worldview of essential importance, particularly for proactively assembling observing data in urban situations. Vehicles normally have no strict imperatives on preparing force and capacity abilities. They can sense occasions (e.g., imaging from roads), process detected information (e.g., perceiving tags), and course messages to different vehicles (e.g., diffusing significant warning to drivers or police operators). In this novel and testing versatile environment, sensors can produce a sheer measure of information, and conventional sensor system approaches for information reporting get to be unfeasible. This article proposes MobEyes, a proficient lightweight backing for proactive urban checking taking into account the essential thought of abusing vehicle versatility to artfully diffuse rundowns about detected information. The reported exploratory/scientific results demonstrate that MobEyes can collect outlines and fabricate an ease appropriated file with sensible fulfillment, great versatility, and restricted overhead

Jakob, Eriksson, Lewis Girod et. al. (2008) [7] In this paper researches an utilization of versatile detecting: recognizing and reporting the surface states of streets. We portray a framework and related calculations to screen this vital common base utilizing a gathering of sensor-prepared vehicles. This framework, which we call the Pothole Patrol (P2), utilizes the characteristic versatility of the taking an interest vehicles, deftly assembling information from vibration and GPS sensors, and preparing the information to survey street surface conditions. We have sent $\mathrm{P} 2$ on 7 taxis running in the Boston range. Utilizing a straightforward machinelearning approach, we demonstrate that we can recognize potholes and other extreme street surface abnormalities from accelerometer information. By means of cautious determination of preparing information and sign components, we have possessed the capacity to manufacture a finder that misidentifies great street fragments as having potholes under $0.2 \%$ of the time. We assess our framework on information from a great many kilometers of taxi drives, and demonstrate that it can effectively recognize various genuine potholes in and around the Boston region. In the wake of bunching to encourage diminish spurious identifications, manual assessment of reported potholes demonstrates that more than $90 \%$ contain street abnormalities needing repair.

Yajie, Ma, Mark Richards et. al. (2008) [8]In this paper, we exhibit a conveyed base taking into account remote sensors system and Grid processing innovation for air contamination checking and mining, which means to grow minimal effort and pervasive sensor systems to gather ongoing, expansive scale and exhaustive ecological information from street movement emanations for air contamination observing in urban environment. The primary informatics challenges in appreciation to building the high-throughput sensor Grid are talked about in this paper. We display a two-layer system structure, a P2P e-Science Grid engineering, and the disseminated information mining calculation as the answers for location the difficulties. We mimicked the framework in 
TinyOS to inspect the operation of every sensor and the systems administration execution. We likewise display the appropriated information mining result to analyze the viability of the calculation.

Giuseppe, Anastasi, Giuseppe Lo Re et. al. (2009) [9] In this paper, observing basic soundness of verifiable legacy structures might be an overwhelming undertaking for structural specialists because of the absence of a prior model for the building solidness, and to the nearness of strict limitations on checking gadget arrangement. This paper provides details regarding the experience maturated amid an undertaking with respect to the outline and usage of an imaginative innovative system for observing basic structures in Sicily, Italy.

Giuseppe, Anastasi, OrazioFarruggiaet. al. (2009) [10] In this paper, this work reports the experience on the outline and organization of a WSN-based framework for checking the profitable cycle of fantastic wine in a Sicilian winery. Other than giving the way to pervasive observing of the developed range, the undertaking depicted here is intended to bolster the maker in guaranteeing the general nature of their generation, as far as exact arranging of mediations in the field, and protection of the put away item. Remote Sensor Networks are utilized as the detecting base of a dispersed framework for the control of a prototypal profitable chain; hubs have been sent both in the field and in the basement, where wine maturing is performed, and information is gathered at a focal unit with a specific end goal to perform surmisings that propose auspicious mediations that protect the grapes' quality.

Artis, Mednis, Girts Strazdinset. al. (2010) [11] In this paper, Road surface investigation including pothole reports is an essential issue for street maintainers and drivers. In this paper we propose a technique for pothole location utilizing portable vehicles outfitted with off the rack receiver and worldwide situating gadgets connected to an on-board PC. The methodology is sufficiently nonexclusive to be stretched out for other sort of occasion identification utilizing diverse sensors also. The vehicles are driving on open roads and measuring pothole prompted sound signs. Our methodology was tried and assessed by genuine tests in a street portion for which we had built up the ground truth previously. The outcomes show pothole discovery with high precision regardless of the foundation commotion and other sound occasions.

A. R., Al-Ali, Imran Zualkernan et. al. (2010) [12] In this paper, an online GPRS-Sensors Array for air contamination observing has been composed, actualized, and tried. The proposed framework comprises of a Mobile Data-Acquisition Unit (Mobile-DAQ) and an altered Internet-Enabled Pollution Monitoring Server (Pollution-Server). The Mobile-DAQ unit incorporates a solitary chip microcontroller, air contamination sensors exhibit, a General Packet Radio Service Modem (GPRS-Modem), and a Global Positioning System Module (GPS-Module). The Pollution-Server is a top of the line PC application server with Internet availability. The Mobile-DAQ unit accumulates air toxins levels (CO, NO2, and SO2), and packs them in an edge with the GPS physical area, time, and date. The casing is in this manner transferred to the GPRS-
Modem and transmitted to the Pollution-Server by means of the general population versatile system. A database server is connected to the Pollution-Server for putting away the poisons level for further utilization by different customers, for example, environment security offices, vehicles enrollment powers, and vacationer and insurance agencies. The PollutionServer is interfaced to Google Maps to show continuous toxins levels and areas in vast metropolitan territories. The framework was effectively tried in the city of Sharjah, UAE. The framework reports constant contaminations level and their area on a 24-h/7-day premise.

Alessandra, De Paola et. al. (2011) [13] In this paper, normal tactile gadgets for measuring natural information are commonly heterogeneous, and present strict vitality limitations; also, they are likely influenced by commotion, and their conduct may differ crosswise over time. Bayesian Networks constitute an appropriate device for pre-handling such information before performing more refined simulated thinking; the methodology proposed here goes for getting the best exchange off amongst execution and expense, by adjusting the working method of the basic tangible gadgets. Besides, self-design of the hubs giving the proof to the Bayesian system is done by method for an on-line multi-target enhancement.

S.C., Hu, Y.C. Wang, Huang et. al. (2011) [14] In this paper, considers a small scale atmosphere checking situation, which more often than not requires conveying a substantial number of sensor hubs to catch ecological data. By misusing vehicular sensor systems (VSNs), it is conceivable to prepare less hubs on autos to accomplish fine-grained observing. In particular, when an auto is moving, it could lead estimations at various areas, in this way gathering bunches of detecting information. To accomplish this objective, this paper proposes VSN design to gather and measure air quality for miniaturized scale atmosphere observing in city zones, where hubs' portability might be wild, (for example, taxis). In the proposed VSN engineering, we address two system related issues:

1) how to adaptively change the reporting rates of versatile hubs to fulfill an objective observing quality with less correspondence overhead and

2) how to misuse deft interchanges to decrease message transmissions. We propose calculations to unravel these two issues and check their exhibitions by reenactments.

What's more, we additionally build up a ZigBee-based model to screen the convergence of carbon dioxide $(\mathrm{CO} 2)$ gas in city zones.

Alessandra, De Paola, Salvatore Gaglio et. al. (2012) [15] In this paper, surrounding Intelligence frameworks are normally portrayed by the utilization of pervasive gear for observing and adjusting the earth as indicated by clients' needs, and to internationally characterized imperatives. Our work depicts the usage of a testbed giving the equipment and programming instruments for the advancement and administration of AmI applications taking into account remote sensor and actuator arranges, whose fundamental objective is vitality putting something aside for worldwide maintainability. An example application is introduced that locations temperature control in a workplace, through a multi-objective 
fluffy controller checking clients' inclinations and vitality utilization.

Srinivas, Devarakonda, ParveenSevusu et. al. (2013) [16] In this paper, customarily, contamination estimations are performed utilizing costly gear at settled areas or committed versatile hardware labs. This is a coarse-grained and costly approach where the contamination estimations are few and far in the middle. In this paper, we show a vehicular-based portable methodology for measuring fine-grained air quality continuously. We propose two practical information cultivating models - one that can be conveyed on open transportation and the second an individual detecting gadget. We introduce preparatory models and talk about execution challenges and early examinations.

\section{CONCLUSION AND FUTURE SCOPE}

Air pollution control in city ranges is one of the fundamental requests of examination in the natural sciences. For each and every traverse the defilement reasons and pollution scattering are divergent, dependent on the assembling consideration, on vehicles activity, on inner sources et cetera, and additionally on the topographical area, temperature of the air, rate and relationship of the wind, and supplementary meteorological conditions components. Innumerable scientific models are used for the portrayal of the associations in the midst of natural security and meteorological elements. An option route to the scientific models is a learning based way that consolidates a few starting points of vision in a dream base. The paper portrays a case find of vision demonstrating in an air contamination utilizing ontologies. In upcoming we will endeavor to use regression models and ontology for city air contamination estimation.

\section{REFERENCES}

[1] J., Fenger, 1999. Urban air quality. Atmospheric environment, 33(29), pp.4877-4900.

[2] Noboru, Yamazoe, Go Sakai, and KengoShimanoe. "Oxide semiconductor gas sensors." Catalysis Surveys from Asia 7, no. 1 (2003): 63-75

[3] Mihaela. Oprea, "A case study of knowledge modelling in an air pollution control decision support system." Ai Communications 18, no. 4 (2005): 293-303.
[4] Michael, Blaschke, Thomas Tille, Phil Robertson, Stefan Mair, Udo Weimar, and Heiko Ulmer. "MEMS gas-sensor array for monitoring the perceived car-cabin air quality." Sensors Journal, IEEE 6, no. 5 (2006): 1298-1308.

[5] H. K. Eminir, Hala Abdel-Galil: Estimation of Fair Pollutant Concentrations fromMeterological Parameters Using Artificial Neural Network, Journal of Electrical engineering, Vol. 57, No. 2, 2006, pp. 105-110

[6] Uichin, Lee, Biao Zhou, Mario Gerla, Eugenio Magistretti, Paolo Bellavista, and Antonio Corradi. "Mobeyes: smart mobs for urban monitoring with a vehicular sensor network." Wireless Communications, IEEE 13, no. 5 (2006): 52-57.

[7] Jakob, Eriksson, Lewis Girod, Bret Hull, Ryan Newton, Samuel Madden, and Hari Balakrishnan. "The pothole patrol: using a mobile sensor network for road surface monitoring." In Proceedings of the 6th international conference on Mobile systems, applications, and services, pp. 29-39. ACM, 2008.

[8] Yajie, Ma, Mark Richards, MoustafaGhanem, YikeGuo, and John Hassard. "Air pollution monitoring and mining based on sensor grid in London." Sensors 8, no. 6 (2008): 3601-3623.

[9] Giuseppe, Anastasi, Giuseppe Lo Re, and Marco Ortolani. "WSNs for structural health monitoring of historical buildings." In Human System Interactions, 2009. HSI'09. 2nd Conference on, pp. 574-579. IEEE, 2009.

[10] Giuseppe, Anastasi, OrazioFarruggia, G. Lo Re, and Michele Ortolani. "Monitoring high-quality wine production using wireless sensor networks." In System Sciences, 2009. HICSS'09. 42nd Hawaii International Conference on, pp. 1-7. IEEE, 2009.

[11] Artis, Mednis, Girts Strazdins, Martins Liepins, AndrisGordjusins, and Leo Selavo. "RoadMic: Road surface monitoring using vehicular sensor networks with microphones." In Networked Digital Technologies, pp. 417-429. Springer Berlin Heidelberg, 2010.

[12] A. R., Al-Ali, Imran Zualkernan, and FadiAloul. "A mobile GPRSsensors array for air pollution monitoring." Sensors Journal, IEEE 10, no. 10 (2010): 1666-1671.

[13] Alessandra,De Paola, Salvatore Gaglio, Giuseppe Lo Re, and Marco Ortolani. "Multi-sensor fusion through adaptive Bayesian networks." In AI* IA 2011: Artificial Intelligence Around Man and Beyond, pp. 360371. Springer Berlin Heidelberg, 2011.

[14] S.C., Hu, Y.C. Wang, Huang, C.Y., Tseng, Y.C.: Measuring air quality in city areas by vehicular wireless sensor networks. J. Syst. Softw. 84(11), 2005-2012 (2011)

[15] Alessandra, De Paola, Salvatore Gaglio, Giuseppe Lo Re, and Marco Ortolani. "Sensor $9 \mathrm{k}$ : A testbed for designing and experimenting with WSN-based ambient intelligence applications." Pervasive and Mobile Computing 8, no. 3 (2012): 448-466.

[16] Srinivas, Devarakonda, ParveenSevusu, Hongzhang Liu, Ruilin Liu, LiviuIftode, and BadriNath. "Real-time air quality monitoring through mobile sensing in metropolitan areas." In Proceedings of the 2nd ACM SIGKDD International Workshop on Urban Computing, p. 15. ACM, 2013. 